\title{
REFERENCES
}

1. Gerhard Ringel, Färbungsprobleme auf Flachen und Graphen, Deutscher Verlag, Berlin, 1959.

2. J. W. T. Youngs, Remarks on the Heawood conjecture (non-orientable case), Bull. Amer. Math. Soc. 74 (1968), 347-353.

University of California, Santa Cruz

\section{GLOBAL ASYMPTOTIC ESTIMATES FOR ELLIPTIC SPECTRAL FUNCTIONS AND EIGENVALUES ${ }^{1}$}

\author{
BY RICHARD BEALS
}

Communicated by Felix Browder, September 25, 1967

The asymptotic behavior of the spectral function of a selfadjoint elliptic operator has been studied extensively; cf. the discussion in [1] and [7]. Recently Agmon and Kannai [2] and Hörmander [8] have obtained error estimates for general operators. Most of this work is concerned with interior estimates for operators with rather smooth coefficients. Here we consider behavior up to the boundary, with minimal assumptions on the coefficients. Details and proofs will appear elsewhere.

Let $a=\sum a_{\alpha}(x) D^{\alpha}$ be an operator of order $m=2 r$ defined on a region $\Omega$ in $R^{n}$. We assume that the boundary $\partial \Omega$ is uniformly regular of class $m+1$ in the sense of [6]. Let $B_{j}=\sum b_{j, \beta}(x) D^{\beta}, j=1,2, \cdots, r$, be an operator of order $m_{j}<m$ defined on an $\epsilon$-neighborhood of $\partial \Omega$. Suppose $0<h \leqq 1$. We assume

(1) $Q$ is uniformly strongly elliptic on $\Omega$.

$(2)_{h}$ The coefficients $a_{\alpha}$ are bounded and measurable on $\Omega$. For $|\alpha|=m$ and $x, y$ in $\Omega$,

$$
\left|a_{\alpha}(y)-a_{\alpha}(x)\right| \leqq c|y-x|^{h} .
$$

(3) ${ }_{h}$ The coefficients $b_{j, \beta}$ and their derivatives of order $\leqq m-m_{j}$ are bounded and continuous on $\Omega$. For $|\beta|=m_{j}$ and $|\gamma|=m-m_{j}$,

$$
\left|D^{\gamma} b_{j, \beta}(y)-D^{\gamma} b_{j, \beta}(x)\right| \leqq c|y-x|^{h} .
$$

\footnotetext{
${ }^{1}$ Research partially supported by the Army Research Office (Durham) under grant DA-ARO-D-31-124-G592.
} 
Let $A$ be the operator in $L^{2}(\Omega)$ which is the restriction of $a$ to the subspace

$$
D(A)=\left\{u \in W^{m, 2}(\Omega) \mid B_{j} u=0 \text { on } \partial \Omega, j=1, \cdots, r\right\} .
$$

Let $a(x, \xi)=\sum_{|\alpha|=m} a_{\alpha}(x) \xi^{\alpha}, \xi \in R^{n}$, and set $a(x)=(2 \pi)^{-n} \int_{a(x, \xi)<1} d \xi$.

Theorem. Suppose $A$ is selfadjoint and semibounded. Let $A=\int \lambda d E_{\lambda}$ be the spectral resolution. Then each $E_{\lambda}$ has a kernel $e_{\lambda}(x, y)$ which is continuous on the closure of $\Omega \times \Omega$. Moreover

$$
\left|e_{\lambda}(x, y)\right| \leqq c_{0} \lambda^{n / m} \text {. }
$$

For $x \in \Omega$, let $\delta(x)$ be the distance from $x$ to $\partial \Omega$. Given $\epsilon>0$,

$$
\begin{aligned}
\mid e_{\lambda}(x, x)- & a(x) \lambda^{n / m} \mid \\
& \leqq c_{1}\left\{\lambda^{n / m-\theta / m+c}+\lambda^{n / m} \exp \left(-c_{2} \lambda^{1 / 2 m} \delta(x)\right)+\delta(x)^{-2 n}\right\},
\end{aligned}
$$

where $\theta=h /(h+3)$.

For $x \in \partial \Omega$, there is a constant $a_{1}(x)$ such that given $\epsilon>0$,

$$
\left|e_{\lambda}(x, x)-a_{1}(x) \lambda^{n / m}\right| \leqq c_{3} \lambda^{n / m-\theta / m+\bullet} \text {. }
$$

The constants $c_{j}$ are independent of $x, y$.

One can give explicit conditions on the system $\left(a, B_{1}, \cdots, B_{r}\right)$ which are necessary and sufficient for $A$ to be selfadjoint and semibounded; cf. [3].

When $\Omega$ is bounded $A$ has a complete set of orthonormal eigenfunctions $\left\{u_{j}\right\}$ corresponding to eigenvalues $\left\{\lambda_{j}\right\}$. Then

$$
e_{\lambda}(x, y)=\sum_{\lambda_{j} \leq \lambda} u_{j}(x) \bar{u}_{j}(y) .
$$

In view of (1) and (2), integration of $e_{\lambda}(x, x)$ over $\Omega$ gives an estimate for the eigenvalues.

Corollary. Suppose $\Omega$ is bounded, let $N(\lambda)$ be the number of eigenvalues of $A$ which are $\leqq \lambda$. For any $\epsilon>0$,

$$
N(\lambda)=\left(\int_{\Omega} a(x) d x\right) \lambda^{n / m}+O\left(\lambda^{n / m-\theta / m+\iota}\right)
$$

as $\lambda \rightarrow \infty$.

For $h=1$ we have $\theta=1 / 4$. The (interior) error estimate in [2], [8] has $\theta=1 / 2$; however the coefficients are assumed to be $C^{\infty}$ and essential use is made of this. In the $C^{\infty}$ case our global estimate (2) can also be obtained with $\theta=1 / 2$. 
An analogue of the Theorem holds without the Hölder continuity assumptions, but with the weaker error estimate $o\left(\lambda^{n / m}\right)$. This gives a correspondingly weaker version of (4), which can also be obtained by a quite different method [4], [5].

\section{REFERENCES}

1. S. Agmon, On kernels, eigenvalues, and eigenfunctions of operators related to elliptic problems, Comm. Pure Appl. Math. 18 (1965), 627-663.

2. S. Agmon and $\mathrm{Y}$. Kannai, On the asymptotic behavior of spectral functions and resolvent kernels of elliptic operators, Israel J. Math. 5 (1967), 1-30.

3. M. S. Agranovic and M. I. Visik, Elliptic problems with a parameter and parabolic problems of general type, Uspehi Mat. Nauk 19 (1963), no. 3, 53-161=Russ. Math Surveys 19 (1964), no. 3, 53-159.

4. R. Beals, On eigenvalue distributions for elliptic operators without smooth coeffcients, Bull. Amer. Math. Soc. 72 (1966), 701-705.

5. - Classes of compact operators and eigenvalue distributions for elliptic operators, Amer. J. Math. 89 (1967).

6. F. E. Browder, On the spectral theory of elliptic operators. I, Math. Ann. 142 (1961), 22-130.

7. - Asymptotic distribution of eigenvalues and eigenfunctions for non-local elliptic boundary value problems. I, Amer. J. Math. 87 (1965), 175-195.

8. L. Hörmander, On the Riesz means of spectral functions and eigenfunction expansions for elliptic operators, (to appear).

University of Chicago 